\title{
Associative learning and sensory neuroplasticity: how does it happen and what is it good for?
}

\author{
John P. McGann \\ Behavioral and Systems Neuroscience, Psychology Department, Rutgers University, Piscataway, New Jersey 08854, USA
}

\begin{abstract}
Historically, the body's sensory systems have been presumed to provide the brain with raw information about the external environment, which the brain must interpret to select a behavioral response. Consequently, studies of the neurobiology of learning and memory have focused on circuitry that interfaces between sensory inputs and behavioral outputs, such as the amygdala and cerebellum. However, evidence is accumulating that some forms of learning can in fact drive stimulus-specific changes very early in sensory systems, including not only primary sensory cortices but also precortical structures and even the peripheral sensory organs themselves. This review synthesizes evidence across sensory modalities to report emerging themes, including the systems' flexibility to emphasize different aspects of a sensory stimulus depending on its predictive features and ability of different forms of learning to produce similar plasticity in sensory structures. Potential functions of this learning-induced neuroplasticity are discussed in relation to the challenges faced by sensory systems in changing environments, and evidence for absolute changes in sensory ability is considered. We also emphasize that this plasticity may serve important nonsensory functions, including balancing metabolic load, regulating attentional focus, and facilitating downstream neuroplasticity.
\end{abstract}

Sensory systems exist to transduce elements of the external world into neural representations of the world that are actionable for other parts of the nervous system. Most neurobiological accounts of sensation model this initial transformation as a fixed process, where the ear is like a microphone and the eye like a camera, each producing an initial neural representation that principally reflects the external stimulus. A given physical stimulus thus always produces roughly the same initial neural representation, presumably allowing it to be recognized quickly and interpreted appropriately. However, in practice the value of using a fixed mapping of physical stimulus onto initial neural representation may be limited. We easily recognize a familiar object seen from a new angle or a favorite song played over a noisy background, circumstances where the peripheral neural representations of the stimulus are actually very different from our previous experience. Outside the laboratory, situations where reoccurrences of a particular stimulus evoke the same pattern of initial neural activity each time are probably extremely rare. In this light, our sensory systems seem designed not to presume that a sensory stimulus will always produce the same neural representation but rather to expect the opposite-that a given stimulus in the world may produce quite different initial neural signals each time it is encountered. Consequently, the sensory system's principal job may be to rapidly recognize ecologically important re-occurring stimuli across these diverse initial representations. This recognition requires that the sensory system act on prior knowledge about the relationships among stimuli in the world, which can only be the result of associative learning. Learning should thus be expected to be accompanied by sensory neuroplasticity, and these changes might need to be as early as the initial neural representation of the sensory stimulus.

The influence of learning on sensation has been appreciated at least since Aristotle's claim that sensory systems change to become like their external stimuli (Aristotle and Shiffman 2011),

Corresponding author: john.mcgann@rutgers.edu

Article is online at http://www.learnmem.org/cgi/doi/10.1101/lm.039636.115. Freely available online through the Learning \& Memory Open Access option. notably including William James's powerful assertion that "every perception is an acquired perception" (James 1890). However, in experimental practice changes derived from sensory plasticity (e.g., the tone sounds quieter) can be challenging to discriminate from changes related to task performance (e.g., the tone sounds the same but evokes the measured behavior less strongly) or stimulus generalization (e.g., the tone sounds quieter, but the subject makes the same behavioral response). This problem is acute for conventional laboratory learning paradigms that teach a subject that an originally neutral stimulus is predictive of threat or reward because any changes in the subject's behavioral response to the stimulus confounds possible changes in perception with anticipation of the learned outcome. Studies of the neurobiology of learning and memory have thus focused on more behaviorally tractable questions, albeit with some notable exceptions. Perhaps as a consequence, "associative learning," where brief training can induce rapid changes in behavior without obvious changes in sensory abilities (Fanselow and Poulos 2005; Weinberger 2007, 2015; Weinberger and Bieszczad 2011), is generally considered separately from "perceptual learning," where extended training is used to induce often modest sensory changes (Gilbert et al. 2001; Brown et al. 2004; Hawkey et al. 2004; Polley et al. 2006; Gilbert and Sigman 2007). However, evidence is accumulating that typical associative learning paradigms (and especially fear conditioning and operant conditioning paradigms) can in fact drive stimulus-specific changes very early in sensory systems, including primary sensory cortices, precortical structures, and even the peripheral sensory organs themselves. Plasticity at such an early stage suggests that the distinction between associative and perceptual learning may thus be largely semantic. To consider the potential functions of the sensory neuroplasticity resulting from these learning paradigms, we must look both at the nature of the changes and their position within the circuit. 


\section{Instances of associative learning-induced sensory plasticity}

It has been known since the early 1980s that associative learning could induce physiological changes in sensory neurons in invertebrates like Aplysia and Hermissenda, where direct connections between sensory and motor systems permit simple physical stimuli to become able to evoke reflexive behaviors (Crow and Alkon 1980; Hawkins et al. 1983; Walters and Byrne 1983). However, associative learning has now been shown to induce sophisticated stimulus-specific neuroplasticity in the mammalian auditory, visual, olfactory, somatosensory, and gustatory systems, which are only indirectly related to behavior. These effects have been observed not only in secondary sensory "association" cortices (Sacco and Sacchetti 2010) but also in primary sensory cortices (Morris et al. 1998; Ohl and Scheich 2005; Polley et al. 2007; Li et al. 2008; Chen et al. 2011; Gdalyahu et al. 2012; Suga 2012; Weinberger 2015), subcortical sensory structures (Edeline and Weinberger 1991a,b, 1992; Cruikshank et al. 1992; Kay and Laurent 1999; Gao and Suga 2000; Doucette et al. 2011; Fletcher 2012), and even primary sensory neurons (Jones et al. 2008; Kass et al. 2013d; Dias and Ressler 2014).

\section{Associative plasticity in the auditory system}

The first evidence of associative learning-related plasticity was reported in the auditory thalamus in 1956 (Galambos et al. 1956). This inspired an extensive series of investigations of learninginduced plasticity in the inferior colliculus, auditory thalamus, and auditory cortices (Ohl and Scheich 2005; Scheich et al. 2011; Schreiner and Polley 2014; Weinberger 2015). In the auditory system, most individual neurons have receptive fields tuned to a single "best frequency" to which they are maximally sensitive and most strongly responsive. Within the cortex and some subcortical structures, neurons are distributed in a rough tonotopic fashion such that best frequencies are mapped onto the spatial location with a gradient from low-to-high frequency across the structure. Plasticity in the auditory system has thus historically been characterized both as changes in the receptive field of single neurons and as remapping of neural populations to alter the number of neurons preferring a given frequency.

The most straightforward studies of auditory plasticity induced by associative learning have relied on single neurons observed before and after conditioning. Early evidence of fear conditioning effects in single cortical neurons was reported in the 1980s (Kraus and Disterhoft 1982; Weinberger et al. 1984; Bakin and Weinberger 1990) when single-unit extracellular recordings of neurons in rodent area A1 often revealed dramatic shifts in their frequency tuning functions such that their best frequencies shifted in the direction of the shock-predictive tone (Fig. 1A). Follow-up experiments recording in the auditory cortex but using either auditory or visual stimuli for fear conditioning confirmed that only auditory-cued fear conditioning altered frequency coding in individual A1 neurons, thus ruling out the possibility that the observed plasticity merely indicated a change in arousal (Bakin et al. 1992), and showing that these effects persist at least $8 \mathrm{wk}$ after learning (Weinberger and Bakin 1998). Analogous experiments trained rats to use sound intensity to localize a target location for reward and proved corresponding changes in the intensity-response functions of neurons in A1 (Polley et al. 2004), while training rats to use azimuth information (direction to the sound source) to perform a task sharpened the azimuth selectivity of A1 neurons (Zhang et al. 2013). These data demonstrate that although frequency tuning is a prominent organizational principle in the auditory system, auditory cortical plasticity permits flexibility in the representation of other stimulus
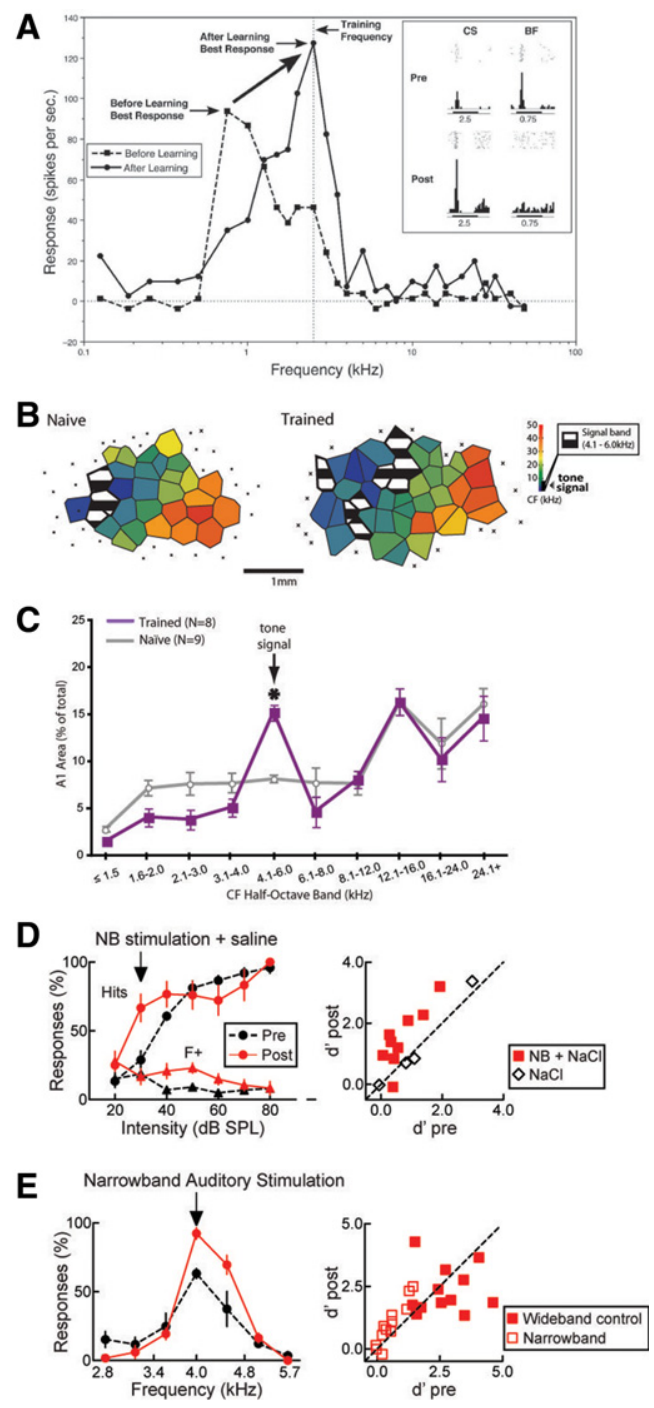

Figure 1. Learning-induced auditory plasticity. $(A)$ Learning-induced change in the preferred frequency of an A1 cortical neuron toward the frequency of the reinforced stimulus. Prior to conditioning, the frequencyresponse curve of this neuron peaked at $\sim 750 \mathrm{~Hz}$ (dashed line), but shifted to $2.5 \mathrm{kHz}$ after conditioning with a $2.5-\mathrm{kHz}$ tone (the CS) paired with shock. The inset shows peristimulus spike histograms for this neuron for the CS frequency and its original $750-\mathrm{Hz}$ best frequency (BF) before and after conditioning. Note the clear change in frequency preference. (Adapted from Weinberger 2007.) (B) Pseudocolored maps showing the electrophysiologically determined frequency preference (characteristic frequency, or CF) for different patches of primary auditory cortex in an experimentally naïve rat (left) versus a rat that had been trained to bar press for reward during presentation of a $5 \mathrm{kHz}$ tone (right). Black and white hatching denotes the regions preferring the frequency band including the training tone. $(C)$ Distribution of cortical area on average across animals as a function of characteristic frequency bands in naïve and trained rats from $B$. ( $B, C$ are adapted from Bieszczad and Weinberger 2010b and used with permission from PNAS.) (D) Changes in behavioral responses on a psychophysical detection task that indicate improved detection of a $4-\mathrm{kHz}$ target stimulus at low stimulus intensities following pairing of the target stimulus with nucleus basalis (NB) stimulation. This is reflected in an increase in Hit rate at low stimulus intensities (left) and an improved $\mathrm{d}^{\prime}$ value (right). (E) Changes in behavioral responses on a psychophysical discrimination task indicating improved discrimination of the CS following narrowband stimulus (left) but not a control wideband stimulus pairing with nucleus basalis stimulation. ( $D, E$ are adapted from Froemke et al. 2013 and used with permission from Nature Publishing Group 2013.) 
dimensions as well. They also show that both aversive and rewarding stimuli can evoke similar, though perhaps not identical, changes in the representation of associated auditory stimuli. Secondary auditory cortices are less well studied but likely exhibit similar learning-induced plasticity (Diamond and Weinberger 1984).

At the population level, pairing a neutral tone CS with shock or reward induces an expansion of the cortical subregions sensitive to the CS frequency (Fig. 1B,C; Rutkowski and Weinberger 2005; Bieszczad and Weinberger 2010a,b) or intensity (Polley et al. 2004, 2006). By varying the degree of water restriction across subjects, Rutkowski and Weinberger (2005) were able to alter the motivational value of a water reward predicted by a tone. They observed that the behaviorally inferred motivation of the rat to bar press for water reward strongly correlated $(r=0.87)$ with the relative size of the area in A1 that responded to that tone's frequency band, while nearby areas representing higher frequencies became proportionately smaller $(r=-0.72)$. If no further training occurs the naturally occurring expansion lasts for weeks, but extinction training, in which the subject learns that the CS no longer predicts the reward, reverses the cortical remapping (Bieszczad and Weinberger 2012). Similar work used classical conditioning in a PET scanner to train human subjects that high- or low-frequency tones predicted an aversive white noise burst and observed frequency-specific changes in auditory-evoked responses in the auditory cortex (Morris et al. 1998), though longer-term imaging experiments in humans have not been possible.

Auditory cortical remapping is now sufficiently well understood to permit some fascinating experiments. Polley et al. (2006) presented rats with a series of auditory cues that varied in both frequency and intensity. For some rats, the frequency variation guided the animal to the target location for reward while in other rats the intensity variation led to the target, but all rats received the same range of auditory stimulation. Remarkably, when the rats were trained to attend to the frequency of the sounds they exhibited a remapping of best frequencies in A1 but no change in local intensity-response functions in A1. Conversely, if they were trained to respond to the sound intensity their intensity-response functions changed but not the tonotopic mapping (Polley et al. 2006). This suggests a more sophisticated plasticity than can be produced by mere temporal conjunction. Indeed, auditory cortical plasticity can selectively enhance cortical responses to tones only when they occur in a particular sequence (Kilgard and Merzenich 2002) or at a particular repetition rate (Bao et al. 2004).

Area A1 is strongly innervated by cholinergic afferents, notably from the nucleus basalis (NB), which can induce strong local plasticity via muscarinic receptors (Ji et al. 2001; Miasnikov et al. 2001; Butt et al. 2009; Froemke et al. 2013). Electrically stimulating the NB during tone presentation alters the tuning of individual neurons (Froemke et al. 2013) and remaps cortex to selectively expand the region representing the corresponding tone (Dimyan and Weinberger 1999) or other acoustic features like frequency sweeps and pulse sequences (Kilgard and Merzenich 1998a,b; Weinberger 1998; Kilgard et al. 2001). Artificially inducing this remapping by pairing NB stimulation with tone presentation results in behavioral changes where rats begin to preferentially respond to the tone whose cortical representation was expanded (Bieszczad et al. 2013) and improve their detection of near or below threshold stimuli (Fig. 1D; Froemke et al. 2013). The circuit-level basis of these population changes is beginning to be understood, with neurophysiological data showing that shock-evoked acetylcholine release can stimulate layer I interneurons in auditory cortex that in turn inhibit inhibitory interneurons in layer II/III, ultimately disinhibiting cortical pyramidal neurons required for auditory fear learning (Letzkus et al. 2011).
How this mechanism translates into persistent population-level remapping of the sensory region and how it interfaces with other neuromodulators like dopamine (Bao et al. 2001; Ilango et al. 2012) and with short-term synaptic dynamics (David et al. 2009) remains to be determined.

The experiments described thus far have all provided evidence of long-term re-mapping of the cortex, with effects that can last days or weeks. However, shorter term plasticity has also been observed. Training ferrets to engage in auditory-cued selective attention tasks can produce rapid changes in the spatiotemporal receptive fields of individual neurons in A1 that persist for hours (Fritz et al. 2003). Similarly, during performance of an auditory discrimination task these neurons can be seen to temporarily alter their adaptation properties to enhance the differences in response between relevant stimuli (Atiani et al. 2014; Shamma and Fritz 2014; Yin et al. 2014). It remains unclear whether these comparatively brief changes during auditory tasks reflect longerterm plasticity that allows the circuit to operate in different taskspecific "modes," or if this short-term response plasticity is mechanistically related to that seen with longer-term remapping at all.

In addition to the remapping of the auditory cortex, associative learning can alter the tuning of neurons in subcortical auditory processing regions, including the medial geniculate body (MGB) of the thalamus. Fear conditioning shifts the best frequency of guinea pig MGB neurons toward that of a threat-predictive auditory cue (Bakin and Weinberger 1990; Cruikshank et al. 1992; Edeline and Weinberger 1992), a shift that lasts for about an hour (Edeline and Weinberger 1991b). This associative plasticity upstream of the lateral nucleus of the amygdala has been noted as potentially playing an underappreciated role in auditory-cued fear learning (Weinberger 2011). Similar changes have been observed following conditioning in the inferior colliculus of the bat (Gao and Suga 2000). To our knowledge, associative learning-induced plasticity has not been tested for in more peripheral auditory structures.

\section{Associative plasticity in the olfactory system}

The olfactory system has played an important role in the study of the sensory effects of associative learning, because it is the only mammalian sensory system where the initial neural representations of external stimuli are experimentally accessible in vivo. Olfactory sensory transduction occurs in olfactory sensory neurons (OSNs) in the olfactory epithelium in the nasal passages. Each OSN expresses just one out of a repertoire of hundreds of odor receptors, and as their axons course back to the brain's olfactory bulb they segregate so that all of the OSNs expressing each individual receptor type project to only one or two specific glomeruli on the surface of the olfactory bulb (Mombaerts et al. 1996; Potter et al. 2001). Visualizing the pattern of synaptic output from OSNs into each of these glomeruli in vivo thus reveals a spatial map where the input to each glomerulus indicates the aggregate activity of OSNs expressing the corresponding receptor. Consequently, the overall pattern of input across glomeruli reliably indicates the identity of the odorant in the nose (Wachowiak and Cohen 2001; Bozza et al. 2004; Soucy et al. 2009). Moreover, the initial convergence of "bottom-up" sensory input from OSNs in the nose with "top-down" projections from sensory cortical and other brain regions occurs in these glomeruli (and not in the thalamus), permitting the effects of descending and neuromodulatory projections to be readily observed. Studies of cortical function and olfactory psychophysics have provided a rich complement to this unusually accessible information on early sensory processing (Li 2014).

In a seminal paper, Jones et al. (2008) used a line of genetargeted mice where all of the OSNs expressing the M71 odor 
receptor could be identified in histological sections of the olfactory epithelium to test the hypothesis that M71 receptor expression in the olfactory epithelium could be altered by extended fear conditioning. In these experiments, they found that fear conditioning these mice with an odorant known to activate M71expressing neurons greatly increased the number of OSNs expressing the M71 receptor within the epithelium 3 wk later (Fig. 2A-C), while mere exposure to that odorant or fear conditioning with a different odorant that does not activate the M71 receptor had no effect. This constituted the first evidence that primary sensory neurons could be affected by associative learning in mammals. Subsequent work from this laboratory has not only replicated this finding but discovered that the descendants of these fear conditioned mice also exhibited elevated numbers of M71 receptorexpressing OSNs and higher behavioral sensitivity to the M71-activating odorant (Dias and Ressler 2014). The epigenetic mechanism of this heritability is incompletely understood but appears to involve changes in DNA methylation in the gene encoding the M71 receptor.

This structural work has been complemented by neurophysiological evidence that the synaptic output of OSN populations exhibits associative, odor-specific enhancement in vivo. In mice expressing the fluorescent exocytosis indicator synaptopHluorin in all mature OSNs (Bozza et al. 2004), odorant-evoked neurotransmitter release from OSN presynaptic terminals was visualized both before and after discriminative olfactory fear conditioning in the same individual mice (Kass et al. 2013d). The behavior of OSN populations that responded selectively to the CS - odorant, which was presented but not paired with shock, was unchanged by $3 \mathrm{~d}$ of discriminative fear conditioning, but OSN populations that selectively responded to the CS+ odor, which did predict an impending footshock, released much more neurotransmitter during CS + presentations after fear conditioning than at preconditioning baseline (Fig. 2D,E). This facilitation increased the sensitivity of these OSNs across odor concentrations such that fear conditioning's effect on the OSN output was comparable with that of a fourfold increase in odorant concentration in naïve animals (Fig. 2F). Surprisingly, OSN populations that responded to both the CS + and CS - odorants (which shared some molecular features and thus both bound to some of the same odor receptors) were only potentiated when responding to the CS+, suggesting that somehow information about the significance of the global representation of the odorant is available even in these specific subpopulations of "primary" sensory neurons. The differential facilitation of single OSN populations when responding to two different odorants and the comparatively brief 3-d interval between the first odorant-shock pairing and the observed plasticity suggests that these physiological changes in OSN activity were not caused by structural changes in the numbers of OSNs. Similarly, facilitated responses have been observed using intrinsic signal imaging (which reflects some combination of presynaptic, postsynaptic, and glial cell metabolism) in olfactory bulb glomeruli
A

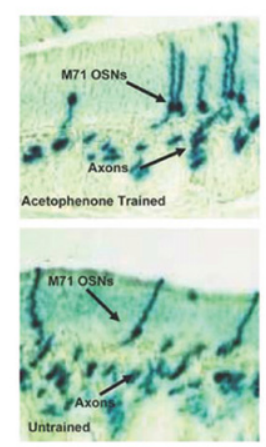

B

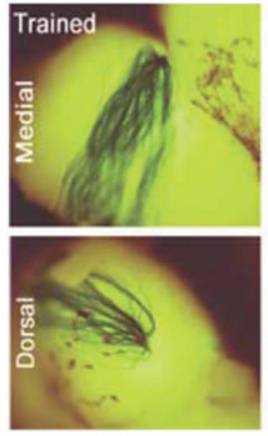

D

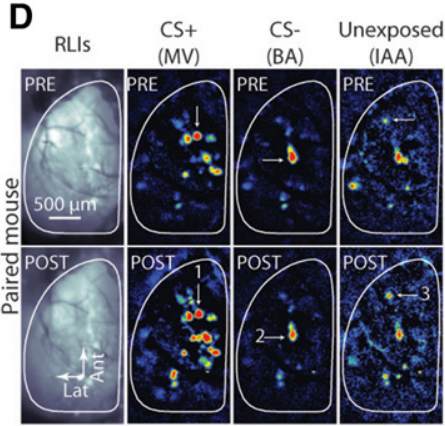

E

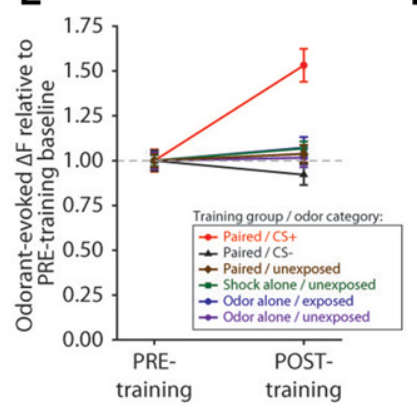

C
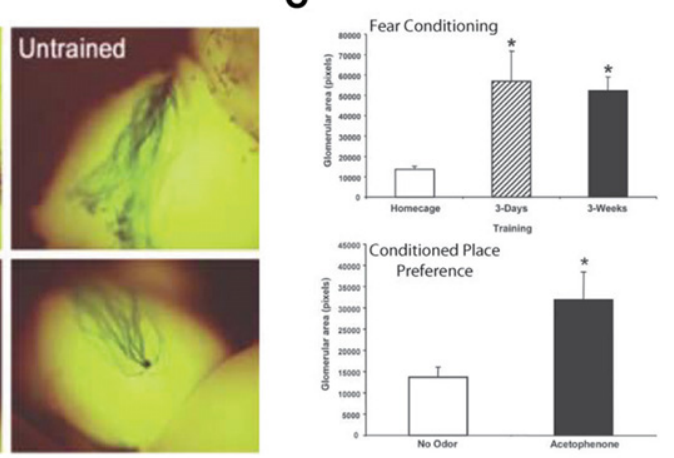

$\mathbf{F}$

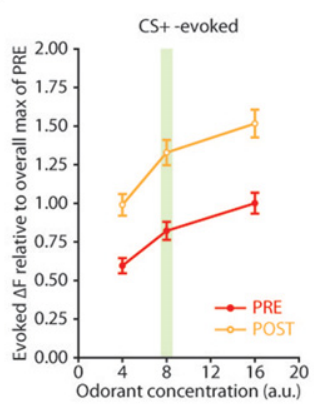

Figure 2. Learning-induced olfactory plasticity in olfactory sensory neurons. $(A)$ Increased numbers of M71 receptor-expressing olfactory sensory neurons in the olfactory epithelium following fear conditioning with the M71-exciting odorant acetophenone (upper) compared with untrained controls (lower). (B) Increased number of axons from M71-expressing olfactory sensory neurons (blue) projecting to the corresponding dorsal and medial glomeruli on the surface of the olfactory bulb following fear conditioning with acetophenone (left) compared with untrained controls (right). ( $C$ ) Increased area of the M71-associated glomerulus following odor-cued fear conditioning (upper) and odor-cued, cocaine-induced, conditioned place preference (lower). ( $A-C$ are adapted from Jones et al. 2008 and used with permission from the Society for Neuroscience 2008.) (D) Mice receiving fear conditioning show enhanced odor-evoked neurotransmitter release from OSNs activated by the shock-predictive (CS + ) odor methyl valerate (MV) after fear conditioning (POST) compared with before fear conditioning (PRE). No change was observed for the shock nonpredictive (CS-) odor butyl acetate (BA) or the unexposed control odor isoamyl acetate (IAA). RLls indicate the resting light image of the olfactory bulb. (E) Summary of overall changes in odor-evoked neurotransmitter release across glomeruli and experimental groups. $(F)$ Enhanced neurotransmitter release across concentrations of the CS+ odor, including those above and below the trained concentration (green bar), indicate a general increase in neural sensitivity to the CS+ odorant. ( $D-F$ are adapted from Kass et al. 2013d and used with permission from the American Association for the Advancement of Science 2013.) 
following odor-cued operant conditioning (Abraham et al. 2014). These physiological changes thus appear to be evoked by both emotionally positive and negative associations and operate in parallel to the changes in OSN numbers observed by Jones et al. (2008) and Dias and Ressler (2014).

Given the plasticity in the initial sensory input to the brain, it is perhaps not surprising that effects of associative learning can be observed at later stages of the olfactory system as well. Within glomeruli, OSNs make synapses onto mitral cells, which serve as the principal output neurons from the olfactory bulb to the piriform cortex. Extracellular recordings from mitral cells in rats during the performance of an operant task have revealed that cellular activity is strongly influenced by learned expectations about whether an odor predicts a reward rather than the mere identity of the odor in the nose (Kay and Laurent 1999; Doucette et al. 2011). Olfactory fear conditioning selectively increases the odor-evoked activity of mitral cells receiving input from OSNs driven by the CS (Fletcher 2012). Similar behavior continues downstream, where olfactory fear conditioning alters the odor response patterns of single neurons in the anterior piriform cortex such that discriminative conditioning between odor mixtures narrows the response tuning of pyramidal neurons while simple conditioning of an odor broadened their tuning (Chen et al. 2011).

In humans, the physiological response to odors is not experimentally accessible in the OSNs or olfactory bulb, but functional magnetic resonance imaging (fMRI) can reveal odor-specific patterns of activity in the piriform cortex. In a fascinating experiment, Li et al. (2008) combined fMRI with psychophysical testing to observe the effects of fear conditioning on pairs of enantiomers, odors that are chemical mirror images of each other and thus usually smell very similar. Prior to aversive conditioning (the human analog of fear conditioning) the two enantiomers in each pair were indiscriminable to subjects and evoked very similar patterns of activity in the piriform cortex. Aversive conditioning was then performed using one odor from one pair of enantiomers as the CS+ and a second odor from a different pair of enantiomers as the $\mathrm{CS}-$. After conditioning, the odor used as the CS+ not only evoked patterns of neural activity in piriform cortex that were now discriminable from its enantiomer counterpart, but the CS+ and its counterpart became perceptually discriminable to the subjects. No changes were observed for the pair used as the $\mathrm{CS}-$. This shows some of the strongest evidence that associative learning induces sensory neuroplasticity in humans with corresponding effects on sensory abilities. Purely psychophysical experiments have also demonstrated that odor-cued aversive conditioning in humans can increase the subject's sensitivity to the shock-predictive odorant (Åhs et al. 2013) and that this facilitation can last at least a few days (Parma et al. 2015).

\section{Associative plasticity in other sensory systems}

Though the most extensive work on associative learning-induced sensory plasticity has been done in the auditory and olfactory systems, mounting evi- dence suggests that it may be ubiquitous across sensory modalities. In the mouse somatosensory cortex, whisker manipulation typically evokes firing across a broad range of cortical neurons within the corresponding cortical barrel. Training the mouse that a particular whisker manipulation predicts an impending shock causes a notable sparsening of these responses, such that fewer neurons respond to the whisker stimulation (Fig. 3A,B) while those that continue to respond (Fig. 3C) exhibit much larger increases in activity (Gdalyahu et al. 2012).

In the rat gustatory cortex (GC), the tastant-evoked spiking behavior of ensembles of cortical neurons changes during conditioned taste aversion learning (Grossman et al. 2008; Moran and Katz 2014). In one study about $30 \%$ of the neurons altered the temporal features of their response to the CS+ tastant (Grossman et al. 2008). Interestingly, these cortical changes were principally observed later in the tastant-evoked response (i.e., after $1 \mathrm{sec}$; see Fig. 3D,E), while such plasticity was evoked throughout the response in simultaneous recordings from the basolateral amygdala (Grossman et al. 2008), suggesting an active shaping of gustatory response during memory retrieval. Neurons exhibiting plasticity in the GC during taste aversion learning partially revert to their original patterns during extinction learning (Moran and Katz 2014). Importantly, gustatory cortical neurons can also exhibit anticipatory responses preceding the unavoidable delivery of a pleasant or unpleasant tastant following classical conditioning (Gardner and Fontanini 2014). The circuit mechanisms underlying this physiological plasticity remain poorly understood, but cholinergic projections to the GC are necessary for the taste aversion learning to occur (Gutierrez et al. 2003; Parkes et al. 2014). As in the auditory system, there is growing evidence that the gustatory thalamus also exhibits associative plasticity and may mediate the learned responses to taste-predictive auditory cues in the GC (Samuelsen et al. 2013).
A
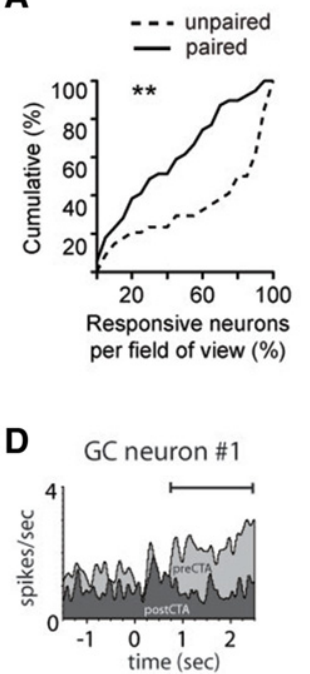

B

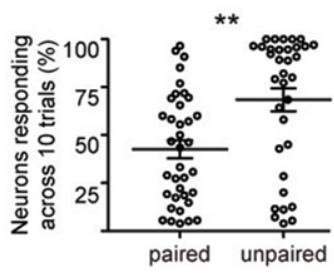

C
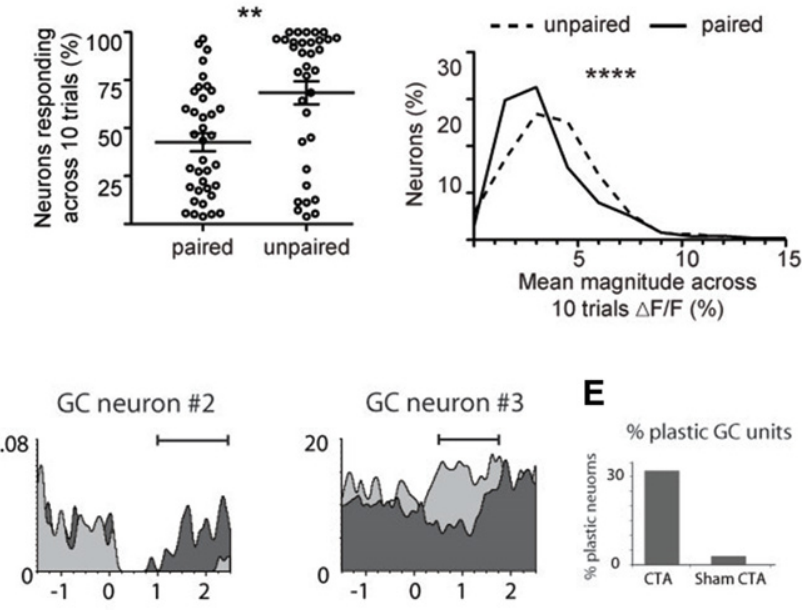

E $\%$ plastic GC units

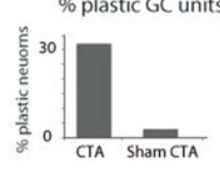

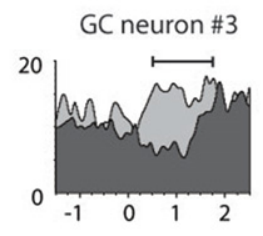

Figure 3. Learning-induced sensory plasticity in the somatosensory and gustatory systems. Whisker-shock fear conditioning reduces the number of individual neurons in the barrel cortex that respond to the shock-predictive whisker movement compared with unpaired controls $(A, B)$, but induces a substantial increase in the size of the whisker-evoked responses in the neurons that do respond (C). ( $A-C$ are adapted from Gdalyahu et al. 2012 and used with permission from Elsevier 2012.) (D) Three examples of individual neurons in the gustatory cortex (GC) whose tastant-evoked responses changed in magnitude and time course following conditioned taste aversion (CTA) learning. The tastant was presented at time zero, and the horizontal bar indicates the time period of statistically significant change. (E) Approximately $30 \%$ of recorded gustatory cortical neurons were changed by conditioned taste aversion learning, while few were changed by a control paradigm. $(D, E$ are adapted from Grossman et al. 2008 and used with permission from the Society for Neuroscience 2008.) 
Visually cued fear conditioning in humans results in increased CS+-evoked BOLD signal not only in the amygdala but also in the extrastriate visual cortex (Knight et al. 1999; Morris et al. 2001). Steady-state visual evoked potentials in visual cortex are selectively amplified for the CS+ following visually cued aversive conditioning in humans (Song and Keil 2014). In rats trained to use visual stimuli to navigate to a hidden platform in a water maze, thalamocortical projections exhibited facilitated long-term potentiation and an increased visual evoked potential was observed in primary visual cortex compared with rats that were exposed to the same visual stimuli when they did not predict the location of the platform (Hager and Dringenberg 2010). In another interesting experiment, rats were fitted with goggles that presented monocular visual cues indicating the effective latency of water reward presentation in an operant task while the activity of single neurons in the deep layers of V1 was recorded (Shuler and Bear 2006). Early in conditioning this activity principally reflected the physical features of the stimulus, but after training many cortical neurons exhibited more complex patterns of activity (including both increase and decreases in firing rate) that persisted after visual stimulus offset and reflected the timing of the anticipated reward. Perhaps most remarkably, in the rat visual system simply associating an auditory cue with a visual cue (in the absence of any explicit reinforcement) is sufficient for the auditory cue to begin to evoke responses in primary visual cortex when the visual cue is later paired with footshock (Headley and Weinberger 2015).

\section{Potential functions}

Despite the robust physiological evidence of sensory plasticity and burgeoning understanding of its circuit-level mechanisms, we still have a limited understanding of how this plasticity is beneficial to the organism. Improved ability to detect stimuli with learned ecological importance would be obviously useful, but in most experiments such improvements are difficult to decouple from purely behavioral responses to the test stimuli (especially when using fear conditioning). Moreover, many potential changes in sensory processing need not actually result in a perceptual change-a tone could produce a larger neural response in A1 without necessarily being perceived as louder, for instance. However, even without outright changes in sensory abilities, sensory plasticity could be useful for directing attention, optimizing memory encoding and retrieval, metabolic savings, or other functions. Some of these functions could also be served by non-associative plasticity evoked by mere exposure to a particular sensory environment (Pienkowski and Eggermont 2009, 2010; Jakkamsetti et al. 2012; Kass et al. 2013b,c; Cadiou et al. 2014), and could potentially use overlapping mechanisms.

\section{Sensory functions}

A common question in interpreting these sensory plasticity studies is why a system capable of improved sensory function would not actually use that potential all the time or for all stimuli. The answer lies in the fact that sensory systems are constantly making tradeoffs. Increased sensitivity risks saturation should the stimulus become stronger, more rigorous discrimination of similar stimuli can impair recognition of the same stimulus on a noisier background, faster discrimination speed often comes at the expense of decreased accuracy, and so forth. Consequently, improvements in sensory function can be surprisingly difficult to define because "better" may depend on the organism's goals or circumstances at that moment. Changes in the neural processing of sensory stimuli could of course reflect absolute improvement in detection or discrimination abilities (Recanzone et al. 1993;
Li et al. 2008; Åhs et al. 2013; Froemke et al. 2013), but these changes may instead reflect retuning of the sensory system's internal parameters to favor discrimination over generalization or sensitivity over dynamic range rather than an expansion in overall physiological abilities. Direct evidence of these tradeoffs after associative learning has not been experimentally accessible, but early auditory exposure to a pure tone that produces a similar expansion of the cortical representation of the corresponding frequency is accompanied by both impaired discrimination of that tone from others and improved discrimination among neighboring tones (Han et al. 2007). Perhaps because of these tradeoffs, there is a growing body of evidence that early neural processing of sensory inputs is actively shaped by task demands (Kay and Laurent 1999; Doucette et al. 2011) and expectations (Headley and Weinberger 2015; Czarnecki et al. in revision), reflecting a plastic, highly flexible processing system.

One of the most straightforward demonstrations of improved sensory function is increased sensitivity to the target stimulus. In a pair of recent odor-cued aversive conditioning studies in human subjects, Lundstrom and colleagues demonstrated a significant reduction in olfactory detection threshold that was specific to the shock-predictive odorant and could last at least several days (Åhs et al. 2013; Parma et al. 2015). Importantly, these findings included clear evidence that the increased sensitivity persisted even after the CS+-evoked skin conductance responses habituated, suggesting that the improved sensory performance was not being confounded by an anticipatory reaction to the shock. This study also found no change in the perceived intensity of the CS+ odorant, suggesting that higher brain regions somehow took the increased sensitivity into account in shaping the ultimate percept. This increased absolute sensitivity is consistent with the neurobiological evidence of increased numbers of CS+responsive OSNs (Jones et al. 2008) and hyperresponsiveness of those OSNs to the CS+ odor (Kass et al. 2013d). Similar improvements in auditory detection have been observed following the induction of A1 plasticity (Fig. 1D) by pairing quiet tones with NB stimulation in rodents performing an auditory-cued operant task (Froemke et al. 2013).

In contrast to a detection task, the discrimination of a target stimulus from similar stimuli may be beneficial or not (Wisniewski et al. 2014), and the stimulus features that guide discrimination and generalization are seemingly determined by the challenges presented during the learning process (Guttman and Kalish 1956; Wisniewski et al. 2009). In effect, setting the correct balance between discrimination and generalization is the key to recognizing a familiar stimulus when encountered from a new perspective or background. Consequently, the mere fact that a stimulus representation has expanded to include more cortical area does not necessarily imply an improvement in discrimination (Talwar and Gerstein 2001; Ohl and Scheich 2005). The studies of Åhs et al. and $\mathrm{Li}$ et al. used an explicit discrimination paradigm, where more than one odor was presented during the conditioning session but only one was paired with shock. This did produce a new ability to discriminate between the shockpredictive odor and its enantiomer (even though that enantiomer was not included in the conditioning paradigm) and clearly demonstrates an increase in absolute discrimination ability. Similarly, in rats trained to discriminate among multiple tone frequencies in an auditory task (Fig. 1E), pairing the target tone with NB stimulation improved discrimination among similar tones (Froemke et al. 2013). After visually cued discriminative aversive conditioning in humans, images of faces that had been paired with an aversive white noise burst were more effectively recognized after being morphed with distractors than faces that had not, though this effect required a period of sleep in between training and testing (Sterpenich et al. 2014). However, in rats, simple 
conditioning that used a single odor during fear conditioning was found to broaden olfactory tuning curves in the piriform cortex, consistent with generalization rather than discrimination, while discriminative conditioning using multiple odors instead narrowed tuning curves, suggesting enhanced discrimination (Chen et al. 2011). This distinction makes ecological sense-in simple conditioning the CS shared many nonolfactory features with the test odor, including being an unnatural odor, being presented at high concentration, and being encountered exclusively in an experimental context, while in discriminative conditioning the inclusion of a CS - odor demonstrated that those nonolfactory features were less predictive of shock than the chemical identity of the odor itself. It remains unknown whether human subjects are equally sensitive to this distinction, but these results suggest that associative learning-induced sensory plasticity may enhance discrimination or reduce it depending on the circumstances.

\section{Nonsensory functions}

As our physiological methods become more capable of observing small neuronal networks (Gdalyahu et al. 2012; Kato et al. 2012; Moran and Katz 2014) and molecularly or anatomically defined cell types (Kass et al. 2013a; Wachowiak et al. 2013), it is growing increasingly clear that "neuroplasticity" can encompass a stunningly broad range of changes in neuronal spiking activity, synaptic connectivity, and neurochemistry, even in the same brain region at the same time. In sensory systems, this realization is reopening classical questions about whether and how specific patterns of neural activity relate to the features of the corresponding physical stimulus as opposed to the predictive utility of that stimulus, its behavioral implications, and other contextual or even cognitive factors (Shuler and Bear 2006; Kass et al. 2013d; Headley and Weinberger 2015). The key questions of sensory neuroplasticity consequently extend beyond the traditional "what new information has been encoded about the stimulus" to envision not just changing neural representations but dynamic neural codes, where the same external stimulus is represented by different firing patterns or even different neurons depending on higher order features like potential meaning, the need for attentional and memory storage resources, and expectation or surprise. Such ideas bring with them homeostatic constraints, like the need to limit the brain's metabolic load, and temporal constraints, where a perception achieved too late will not be actionable for the organism. Learning-induced changes in sensory systems may very well serve these goals rather than traditional sensory functions.

Neural computation is metabolically expensive, with action potential firing and especially synaptic signaling consuming the largest part of the brain's energy budget, including ATP and oxygen consumption (Harris and Attwell 2012). Reductions in firing rate or in the number of neurons responding to a stimulus (Gdalyahu et al. 2012; Kass et al. 2013b) may thus reflect an optimization of stimulus representations according to metabolic constraints, while changes in spike timing or synchrony (Bao et al. 2004; Weinberger et al. 2013; Kay 2015) or increases in neural response to one stimulus balanced by decreased response to other stimuli (Froemke et al. 2013) present potentially metabolically neutral mechanisms for encoding stimulus importance. In this light the large increases in stimulus-evoked synaptic activity (Kass et al. 2013d) and action potential firing rates (Bakin and Weinberger 1990; Polley et al. 2004) after conditioning constitute an investment of significant resources that presumably serves an important purpose.

A critical function of early sensory systems is the allocation of limited attentional resources, as reflected in both neural processing and the selection of stimulus sampling behaviors. Some of the earliest work on classical conditioning-induced changes in sensory processing noted that even in an organism as simple as Aplysia, heightened sensory responses to a conditioned stimulus could facilitate attention and the selection of an ecologically appropriate response (Walters and Byrne 1983). In mammals, attentional systems exhibit an almost paradoxical system of preattentive processing, where many stimuli are superficially evaluated in parallel and across sensory modalities to select the next focus of attention for in-depth serial evaluation (Treisman 2006). The purpose of the usually heightened neural response to threat- or reward-predictive stimuli may not be to improve the sensory processing of these stimuli per se but to increase the likelihood that those stimuli attract top-down attention when they do occur. While selective attention has proved difficult to study in animal models, there is considerable recent evidence that aversive conditioning in humans does indeed produce an attentional bias such that threat-predictive stimuli tend to attract and hold attention (Croy et al. 2010; Felmingham et al. 2011). This bias improves preattentive processing by enhancing detection of masked target stimuli (Armony and Dolan 2002; Kleim et al. 2012), possibly through the conditioned facilitation of activity in sensory regions. Computational efforts to reconstruct the sensory stimulus from the activity it elicits in sensory cortex have demonstrated that changes in attentional and motivational state can notably influence the balance among sensory features in its neural representation (Mesgarani et al. 2009).

Finally, the usually increased neural response to threatpredictive stimuli may serve to facilitate learning and memory itself. The greater activity levels associated with threat- or rewardpredictive stimuli are often similar to those evoked by higher intensity, more salient versions of the same sensory stimulus (e.g., the larger odor-evoked input to the brain after olfactory fear conditioning is similar to the input evoked by a fourfold stronger presentation of the same odor; see Kass et al. 2013d), which generally are learned about more quickly than weaker stimuli. Many forms of synaptic plasticity occur more strongly when presynaptic neurons are firing more quickly or more synchronously (Caporale and Dan 2008; Edeline 2012), suggesting that heightened sensory responses could directly increase the rate of learning about the stimulus in a virtuous cycle. Though the details may depend on the location and stimulation paradigm, there is evidence that some forms of sensory plasticity emerge and then revert, either over time (Edeline and Weinberger 1991b) or with continued training on an associative learning task (Takahashi et al. 2011). This plasticity may thus facilitate the transfer of information about important stimuli to downstream regions (Jeanne et al. 2013) or potentially expand the range of local stimulus processing algorithms, of which the most effective subset are selected for retention (Takahashi et al. 2013). Following learning, the larger stimulus response could also facilitate the retrieval of stimulusassociated memories and selection of stimulus-appropriate behaviors (Znamenskiy and Zador 2013).

\section{Conclusions}

Associative learning can induce dramatic stimulus-specific plasticity in both the behavior of individual sensory neurons and the macroscopic remapping of sensory regions, including auditory, olfactory, somatosensory, gustatory, and visual systems. These effects are best understood in primary sensory cortices but also occur in precortical structures and even as early as the primary sensory neurons themselves. Mechanisms of this plasticity remain poorly understood, but clearly include a role for neuromodulators (notably acetylcholine) as a trigger to evoke plasticity during or after learning. The function of these forms of plasticity may be to improve detection or discrimination of critical stimuli or to 
enable these stimuli to better engage attentional and learning circuitry.

In the auditory system, there has been considerable debate about the best way to conceptualize associative learning-induced cortical and subcortical plasticity. This debate has evolved along with our changing understanding of the auditory cortex's function, initially emphasizing changes in frequency coding (Bakin and Weinberger 1990), expanding to emphasize spectrotemporal and spatial elements of the auditory stimuli (Polley et al. 2006; Froemke et al. 2013; Zhang et al. 2013; Schreiner and Polley 2014), and more recently emphasizing more cognitive models of cortical function like categorization of stimuli (Scheich et al. 2011) and dynamic, task-dependent reshaping of representations (Shamma and Fritz 2014; Yin et al. 2014). The more recent observations of learning-related plasticity in the olfactory, gustatory, and somatosensory systems have similarly been interpreted in light of the current understanding of each sensory structure's purpose. The theme of this evolution is ultimately teleological: as we develop new ideas about what each system is for, we find corresponding explanations for why associative plasticity might be useful. However, regardless of the distinct neurobiological details associated with transducing and analyzing the physical stimulus for each modality, these diverse sensory systems share the challenge of detecting, recognizing, and cueing the organism's response to a changing stimulus in a changing world. By comparing the effects of associative learning across sensory systems we may find thus insight not only into shared neuroplasticity motifs but also into the basic functions of the sensory systems themselves.

\section{Acknowledgments}

We thank Kasia Bieszczad, Cindy Fast, Michelle Rosenthal, and Marley Kass for helpful discussion. This paper was supported by grants from NIMH (R01 MH101293) and NIDCD (R01 DC013090).

\section{References}

Abraham NM, Vincis R, Lagier S, Rodriguez I, Carleton A. 2014. Long term functional plasticity of sensory inputs mediated by olfactory learning. Elife 3: e02109.

Åhs F, Miller SS, Gordon AR, Lundstrom JN. 2013. Aversive learning increases sensory detection sensitivity. Biol Psychol 92: 135-141.

Aristotle. 2011. De anima. (Translated by M. Shiffman) Focus Publishing/ R. Pullins Co., Newburyport, MA.

Armony JL, Dolan RJ. 2002. Modulation of spatial attention by fearconditioned stimuli: an event-related fMRI study. Neuropsychologia 40: $817-826$.

Atiani S, David SV, Elgueda D, Locastro M, Radtke-Schuller S, Shamma SA, Fritz JB. 2014. Emergent selectivity for task-relevant stimuli in higher-order auditory cortex. Neuron 82: 486-499.

Bakin JS, Weinberger NM. 1990. Classical conditioning induces CS-specific receptive field plasticity in the auditory cortex of the guinea pig. Brain Res 536: 271-286.

Bakin JS, Lepan B, Weinberger NM. 1992. Sensitization induced receptive field plasticity in the auditory cortex is independent of CS-modality. Brain Res 577: 226-235.

Bao S, Chan VT, Merzenich MM. 2001. Cortical remodelling induced by activity of ventral tegmental dopamine neurons. Nature 412: 79-83.

Bao S, Chang EF, Woods J, Merzenich MM. 2004. Temporal plasticity in the primary auditory cortex induced by operant perceptual learning. Nat Neurosci 7: 974-981.

Bieszczad KM, Weinberger NM. 2010a. Learning strategy trumps motivational level in determining learning-induced auditory cortical plasticity. Neurobiol Learn Mem 93: 229-239.

Bieszczad KM, Weinberger NM. 2010b. Representational gain in cortical area underlies increase of memory strength. Proc Natl Acad Sci 107: $3793-3798$.

Bieszczad KM, Weinberger NM. 2012. Extinction reveals that primary sensory cortex predicts reinforcement outcome. Eur J Neurosci 35: $598-613$.
Bieszczad KM, Miasnikov AA, Weinberger NM. 2013. Remodeling sensory cortical maps implants specific behavioral memory. Neuroscience 246: 40-51.

Bozza T, McGann JP, Mombaerts P, Wachowiak M. 2004. In vivo imaging of neuronal activity by targeted expression of a genetically encoded probe in the mouse. Neuron 42: 9-21.

Brown M, Irvine DR, Park VN. 2004. Perceptual learning on an auditory frequency discrimination task by cats: association with changes in primary auditory cortex. Cereb Cortex 14: 952-965.

Butt AE, Chavez CM, Flesher MM, Kinney-Hurd BL, Araujo GC, Miasnikov AA, Weinberger NM. 2009. Association learning-dependent increases in acetylcholine release in the rat auditory cortex during auditory classical conditioning. Neurobiol Learn Mem 92: 400-409.

Cadiou H, Aoude I, Tazir B, Molinas A, Fenech C, Meunier N, Grosmaitre X. 2014. Postnatal odorant exposure induces peripheral olfactory plasticity at the cellular level. J Neurosci 34: 4857-4870.

Caporale N, Dan Y. 2008. Spike timing-dependent plasticity: a Hebbian learning rule. Annu Rev Neurosci 31: 25-46.

Chen CF, Barnes DC, Wilson DA. 2011. Generalized vs. stimulus-specific learned fear differentially modifies stimulus encoding in primary sensory cortex of awake rats. J Neurophysiol 106: 3136-3144.

Crow TJ, Alkon DL. 1980. Associative behavioral modification in hermissenda: cellular correlates. Science 209: 412-414.

Croy I, Schellong J, Joraschky P, Hummel T. 2010. PTSD, but not childhood maltreatment, modifies responses to unpleasant odors. Int $J$ Psychophysiol 75: 326-331.

Cruikshank SJ, Edeline JM, Weinberger NM. 1992. Stimulation at a site of auditory-somatosensory convergence in the medial geniculate nucleus is an effective unconditioned stimulus for fear conditioning. Behav Neurosci 106: 471-483.

David SV, Mesgarani N, Fritz JB, Shamma SA. 2009. Rapid synaptic depression explains nonlinear modulation of spectro-temporal tuning in primary auditory cortex by natural stimuli. J Neurosci 29: 3374-3386.

Diamond DM, Weinberger NM. 1984. Physiological plasticity of single neurons in auditory cortex of the cat during acquisition of the pupillary conditioned response: II. Secondary field (AII). Behav Neurosci 98: $189-210$.

Dias BG, Ressler KJ. 2014. Parental olfactory experience influences behavior and neural structure in subsequent generations. Nat Neurosci 17: 89-96.

Dimyan MA, Weinberger NM. 1999. Basal forebrain stimulation induces discriminative receptive field plasticity in the auditory cortex. Behav Neurosci 113: 691-702.

Doucette W, Gire DH, Whitesell J, Carmean V, Lucero MT, Restrepo D. 2011. Associative cortex features in the first olfactory brain relay station. Neuron 69: 1176-1187.

Edeline JM. 2012. Beyond traditional approaches to understanding the functional role of neuromodulators in sensory cortices. Front Behav Neurosci 6: 45

Edeline JM, Weinberger NM. 1991a. Subcortical adaptive filtering in the auditory system: associative receptive field plasticity in the dorsal medial geniculate body. Behav Neurosci 105: 154-175.

Edeline JM, Weinberger NM. 1991b. Thalamic short-term plasticity in the auditory system: associative returning of receptive fields in the ventral medial geniculate body. Behav Neurosci 105: 618-639.

Edeline JM, Weinberger NM. 1992. Associative retuning in the thalamic source of input to the amygdala and auditory cortex: receptive field plasticity in the medial division of the medial geniculate body. Behav Neurosci 106: 81-105.

Fanselow MS, Poulos AM. 2005. The neuroscience of mammalian associative learning. Annu Rev Psychol 56: 207-234.

Felmingham KL, Rennie C, Manor B, Bryant RA. 2011. Eye tracking and physiological reactivity to threatening stimuli in posttraumatic stress disorder. J Anxiety Disord 25: 668-673.

Fletcher ML. 2012. Olfactory aversive conditioning alters olfactory bulb mitral/tufted cell glomerular odor responses. Front Syst Neurosci 6: 16.

Fritz J, Shamma S, Elhilali M, Klein D. 2003. Rapid task-related plasticity of spectrotemporal receptive fields in primary auditory cortex. Nat Neurosci 6: 1216-1223.

Froemke RC, Carcea I, Barker AJ, Yuan K, Seybold BA, Martins AR, Zaika N, Bernstein H, Wachs M, Levis PA, et al. 2013. Long-term modification of cortical synapses improves sensory perception. Nat Neurosci 16: 79-88.

Galambos R, Sheatz G, Vernier VG. 1956. Electrophysiological correlates of a conditioned response in cats. Science 123: $376-377$.

Gao E, Suga N. 2000. Experience-dependent plasticity in the auditory cortex and the inferior colliculus of bats: role of the corticofugal system. Proc Natl Acad Sci 97: 8081-8086.

Gardner MP, Fontanini A. 2014. Encoding and tracking of outcome-specific expectancy in the gustatory cortex of alert rats. J Neurosci 34: 13000-13017. 
Gdalyahu A, Tring E, Polack PO, Gruver R, Golshani P, Fanselow MS, Silva AJ, Trachtenberg JT. 2012. Associative fear learning enhances sparse network coding in primary sensory cortex. Neuron 75: 121-132.

Gilbert CD, Sigman M. 2007. Brain states: top-down influences in sensory processing. Neuron 54: 677-696.

Gilbert CD, Sigman M, Crist RE. 2001. The neural basis of perceptual learning. Neuron 31: 681-697.

Grossman SE, Fontanini A, Wieskopf JS, Katz DB. 2008. Learning-related plasticity of temporal coding in simultaneously recorded amygdala-cortical ensembles. J Neurosci 28: 2864-2873.

Gutierrez R, Rodriguez-Ortiz CJ, De La Cruz V, Nunez-Jaramillo L, Bermudez-Rattoni F. 2003. Cholinergic dependence of taste memory formation: evidence of two distinct processes. Neurobiol Learn Mem 80: $323-331$.

Guttman N, Kalish HI. 1956. Discriminability and stimulus generalization. J Exp Psychol 51: 79-88.

Hager AM, Dringenberg HC. 2010. Training-induced plasticity in the visual cortex of adult rats following visual discrimination learning. Learn Mem 17: $394-401$

Han YK, Köver H, Insanally MN, Semerdjian JH, Bao S. 2007. Early experience impairs perceptual discrimination. Nat Neurosci 10: 1191-1197.

Harris JJ, Attwell D. 2012. The energetics of CNS white matter. J Neurosci 32: $356-371$.

Hawkey DJ, Amitay S, Moore DR. 2004. Early and rapid perceptual learning. Nat Neurosci 7: 1055-1056.

Hawkins RD, Abrams TW, Carew TJ, Kandel ER. 1983. A cellular mechanism of classical conditioning in Aplysia: activity-dependent amplification of presynaptic facilitation. Science 219: 400-405.

Headley DB, Weinberger NM. 2015. Relational associative learning induces cross-modal plasticity in early visual cortex. Cereb Cortex 25: $1306-1318$.

Ilango A, Shumake J, Wetzel W, Scheich H, Ohl FW. 2012. The role of dopamine in the context of aversive stimuli with particular reference to acoustically signaled avoidance learning. Front Neurosci 6: 132.

Jakkamsetti V, Chang KQ, Kilgard MP. 2012. Reorganization in processing of spectral and temporal input in the rat posterior auditory field induced by environmental enrichment. J Neurophysi 107: 1457-1475.

James W. 1890. The principles of psychology. Henry Holt and Company, New York.

Jeanne JM, Sharpee TO, Gentner TQ. 2013. Associative learning enhances population coding by inverting interneuronal correlation patterns. Neuron 78: 352-363.

Ji W, Gao E, Suga N. 2001. Effects of acetylcholine and atropine on plasticity of central auditory neurons caused by conditioning in bats. J Neurophysiol 86: 211-225.

Jones SV, Choi DC, Davis M, Ressler KJ. 2008. Learning-dependent structural plasticity in the adult olfactory pathway. J Neurosci 28: 13106-13111.

Kass MD, Moberly AH, McGann JP. 2013a. Spatiotemporal alterations in primary odorant representations in olfactory marker protein knockout mice. PLoS One 8: e61431.

Kass MD, Moberly AH, Rosenthal MC, Guang SA, McGann JP. 2013b. Odor-specific, olfactory marker protein-mediated sparsening of primary olfactory input to the brain after odor exposure. J Neurosci 33: $6594-6602$.

Kass MD, Pottackal J, Turkel DJ, McGann JP. 2013c. Changes in the neural representation of odorants after olfactory deprivation in the adult mouse olfactory bulb. Chem Senses 38: 77-89.

Kass MD, Rosenthal MC, Pottackal J, McGann JP. 2013d. Fear learning enhances neural responses to threat-predictive sensory stimuli. Science 342: 1389-1392.

Kato HK, Chu MW, Isaacson JS, Komiyama T. 2012. Dynamic sensory representations in the olfactory bulb: modulation by wakefulness and experience. Neuron 76: 962-975.

Kay LM. 2015. Olfactory system oscillations across phyla. Curr Opin Neurobiol 31: 141-147.

Kay LM, Laurent G. 1999. Odor- and context-dependent modulation of mitral cell activity in behaving rats. Nat Neurosci 2: 1003-1009.

Kilgard MP, Merzenich MM. 1998a. Cortical map reorganization enabled by nucleus basalis activity. Science 279: 1714-1718.

Kilgard MP, Merzenich MM. 1998b. Plasticity of temporal information processing in the primary auditory cortex. Nat Neurosci 1: 727-731.

Kilgard MP, Merzenich MM. 2002. Order-sensitive plasticity in adult primary auditory cortex. Proc Natl Acad Sci 99: 3205-3209.

Kilgard MP, Pandya PK, Vazquez J, Gehi A, Schreiner CE, Merzenich MM. 2001. Sensory input directs spatial and temporal plasticity in primary auditory cortex. J Neurophysiol 86: 326-338.

Kleim B, Ehring T, Ehlers A. 2012. Perceptual processing advantages for trauma-related visual cues in post-traumatic stress disorder. Psychol Med 42: 173-181.
Knight DC, Smith CN, Stein EA, Helmstetter FJ. 1999. Functional MRI of human Pavlovian fear conditioning: patterns of activation as a function of learning. Neuroreport 10: 3665-3670.

Kraus N, Disterhoft JF. 1982. Response plasticity of single neurons in rabbit auditory association cortex during tone-signalled learning. Brain Res 246: $205-215$.

Letzkus JJ, Wolff SB, Meyer EM, Tovote P, Courtin J, Herry C, Lüthi A. 2011. A disinhibitory microcircuit for associative fear learning in the auditory cortex. Nature 480: 331-335.

Li W. 2014. Learning to smell danger: acquired associative representation of threat in the olfactory cortex. Front Behav Neurosci 8: 98.

Li W, Howard JD, Parrish TB, Gottfried JA. 2008. Aversive learning enhances perceptual and cortical discrimination of indiscriminable odor cues. Science 319: 1842-1845.

Mesgarani N, David SV, Fritz JB, Shamma SA. 2009. Influence of context and behavior on stimulus reconstruction from neural activity in primary auditory cortex. J Neurophysiol 102: 3329-3339.

Miasnikov AA, McLin D III, Weinberger NM. 2001. Muscarinic dependence of nucleus basalis induced conditioned receptive field plasticity. Neuroreport 12: $1537-1542$.

Mombaerts P, Wang F, Dulac C, Chao SK, Nemes A, Mendelsohn M, Edmondson J, Axel R. 1996. Visualizing an olfactory sensory map. Cell 87: $675-686$

Moran A, Katz DB. 2014. Sensory cortical population dynamics uniquely track behavior across learning and extinction. J Neurosci 34: $1248-1257$.

Morris JS, Friston KJ, Dolan RJ. 1998. Experience-dependent modulation of tonotopic neural responses in human auditory cortex. Proc Biol Sci 265: $649-657$.

Morris JS, Buchel C, Dolan RJ. 2001. Parallel neural responses in amygdala subregions and sensory cortex during implicit fear conditioning. Neuroimage 13: $1044-1052$.

Ohl FW, Scheich H. 2005. Learning-induced plasticity in animal and human auditory cortex. Curr Opin Neurobiol 15: 470-477.

Parkes SL, De la Cruz V, Bermúdez-Rattoni F, Coutureau E, Ferreira G. 2014. Differential role of insular cortex muscarinic and NMDA receptors in one-trial appetitive taste learning. Neurobiol Learn Mem 116: 112-116.

Parma V, Ferraro S, Miller SS, Ảhs F, Lundström JN. 2015. Enhancement of odor sensitivity following repeated odor and visual fear conditioning. Chem Senses 40: 497-506.

Pienkowski M, Eggermont JJ. 2009. Long-term, partially-reversible reorganization of frequency tuning in mature cat primary auditory cortex can be induced by passive exposure to moderate-level sounds. Hear Res 257: 24-40.

Pienkowski M, Eggermont JJ. 2010. Passive exposure of adult cats to moderate-level tone pip ensembles differentially decreases AI and AII responsiveness in the exposure frequency range. Hear Res $\mathbf{2 6 8}$ : $151-162$.

Polley DB, Heiser MA, Blake DT, Schreiner CE, Merzenich MM. 2004. Associative learning shapes the neural code for stimulus magnitude in primary auditory cortex. Proc Natl Acad Sci 101: 16351-16356.

Polley DB, Steinberg EE, Merzenich MM. 2006. Perceptual learning directs auditory cortical map reorganization through top-down influences. J Neurosci 26: 4970-4982.

Polley DB, Read HL, Storace DA, Merzenich MM. 2007. Multiparametric auditory receptive field organization across five cortical fields in the albino rat. J Neurophysiol 97: 3621-3638.

Potter SM, Zheng C, Koos DS, Feinstein P, Fraser SE, Mombaerts P. 2001. Structure and emergence of specific olfactory glomeruli in the mouse. J Neurosci 21: 9713-9723.

Recanzone GH, Schreiner CE, Merzenich MM. 1993. Plasticity in the frequency representation of primary auditory cortex following discrimination training in adult owl monkeys. J Neurosci 13: 87-103.

Rutkowski RG, Weinberger NM. 2005. Encoding of learned importance of sound by magnitude of representational area in primary auditory cortex. Proc Natl Acad Sci 102: 13664-13669.

Sacco T, Sacchetti B. 2010. Role of secondary sensory cortices in emotional memory storage and retrieval in rats. Science 329: 649-656.

Samuelsen CL, Gardner MPH, Fontanini A. 2013. Thalamic contribution to cortical processing of taste and expectation. J Neurosci 33: 1815-1827.

Scheich H, Brechmann A, Brosch M, Budinger E, Ohl FW, Selezneva E, Stark H, Tischmeyer W, Wetzel W. 2011. Behavioral semantics of learning and crossmodal processing in auditory cortex: the semantic processor concept. Hear Res 271: 3-15.

Schreiner CE, Polley DB. 2014. Auditory map plasticity: diversity in causes and consequences. Curr Opin Neurobiol 24: 143-156.

Shamma S, Fritz J. 2014. Adaptive auditory computations. Curr Opin Neurobiol 25: 164-168.

Shuler MG, Bear MF. 2006. Reward timing in the primary visual cortex. Science 311: 1606-1609. 
Song I, Keil A. 2014. Differential classical conditioning selectively heightens response gain of neural population activity in human visual cortex. Psychophysiology 51: 1185-1194.

Soucy ER, Albeanu DF, Fantana AL, Murthy VN, Meister M. 2009. Precision and diversity in an odor map on the olfactory bulb. Nat Neurosci 12: 210-220.

Sterpenich V, Piguet C, Desseilles M, Ceravolo L, Gschwind M, Van De Ville D, Vuilleumier P, Schwartz S. 2014. Sleep sharpens sensory stimulus coding in human visual cortex after fear conditioning. Neuroimage 100: 608-618.

Suga N. 2012. Tuning shifts of the auditory system by corticocortical and corticofugal projections and conditioning. Neurosci Biobehav Rev 36: 969-988.

Takahashi H, Yokota R, Funamizu A, Kose H, Kanzaki R. 2011. Learning-stage-dependent, field-specific, map plasticity in the rat auditory cortex during appetitive operant conditioning. Neuroscience 199: $243-258$.

Takahashi H, Yokota R, Kanzaki R. 2013. Response variance in functional maps: neural Darwinism revisited. PLoS One 8: e68705.

Talwar SK, Gerstein GL. 2001. Reorganization in awake rat auditory cortex by local microstimulation and its effect on frequency-discrimination behavior. J Neurophysiol 86: 1555-1572.

Treisman A. 2006. How the deployment of attention determines what we see. Vis $\operatorname{Cogn}$ 14: 411-443.

Wachowiak M, Cohen LB. 2001. Representation of odorants by receptor neuron input to the mouse olfactory bulb. Neuron 32: 723-735.

Wachowiak M, Economo MN, Díaz-Quesada M, Brunert D, Wesson DW, White JA, Rothermel M. 2013. Optical dissection of odor information processing in vivo using GCaMPs expressed in specified cell types of the olfactory bulb. J Neurosci 33: 5285-5300.

Walters ET, Byrne JH. 1983. Associative conditioning of single sensory neurons suggests a cellular mechanism for learning. Science 219: 405-408.

Weinberger NM. 1998. Tuning the brain by learning and by stimulation of the nucleus basalis. Trends Cogn Sci 2: 271-273.

Weinberger NM. 2007. Associative representational plasticity in the auditory cortex: a synthesis of two disciplines. Learn Mem 14: 1-16.
Weinberger NM. 2011. The medial geniculate, not the amygdala, as the root of auditory fear conditioning. Hear Res 274: 61-74.

Weinberger NM. 2015. New perspectives on the auditory cortex: learning and memory. Handb Clin Neurol 129: 117-147.

Weinberger NM, Bakin JS. 1998. Learning-induced physiological memory in adult primary auditory cortex: receptive fields plasticity, model, and mechanisms. Audiol Neurootol 3: 145-167.

Weinberger NM, Bieszczad KM. 2011. Introduction: from traditional fixed cortical sensationism to contemporary plasticity of primary sensory cortical representations. In Neurobiology of sensation and reward, (ed. Gottfried JA), Boca Raton, FL.

Weinberger NM, Hopkins W, Diamond DM. 1984. Physiological plasticity of single neurons in auditory cortex of the cat during acquisition of the pupillary conditioned response: I. Primary field (AI). Behav Neurosci 98: $171-188$.

Weinberger NM, Miasnikov AA, Bieszczad KM, Chen JC. 2013. $\gamma$ Band plasticity in sensory cortex is a signature of the strongest memory rather than memory of the training stimulus. Neurobiol Learn Mem 104: 49-63.

Wisniewski MG, Church BA, Mercado E III. 2009. Learning-related shifts in generalization gradients for complex sounds. Learn Behav 37: $325-335$.

Wisniewski MG, Liu EH, Church BA, Mercado E III. 2014. Learning to discriminate frequency modulation rate can benefit and worsen pitch acuity. J Acoust Soc Am 135: EL55-EL60.

Yin P, Fritz JB, Shamma SA. 2014. Rapid spectrotemporal plasticity in primary auditory cortex during behavior. J Neurosci 34: 4396-4408.

Zhang Y, Zhao Y, Zhu X, Sun X, Zhou X. 2013. Refining cortical representation of sound azimuths by auditory discrimination training. I Neurosci 33: 9693-9698.

Znamenskiy P, Zador AM. 2013. Corticostriatal neurons in auditory cortex drive decisions during auditory discrimination. Nature 497: 482-485.

Received July 27, 2015; accepted in revised form September 3, 2015. 


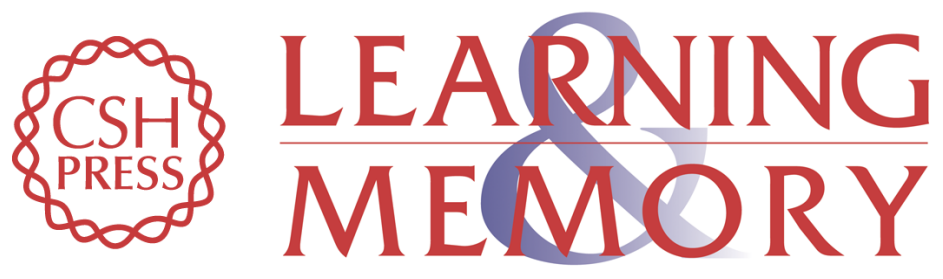

\section{Associative learning and sensory neuroplasticity: how does it happen and what is it good for?}

John P. McGann

Learn. Mem. 2015, 22:

Access the most recent version at doi:10.1101//m.039636.115

References This article cites 118 articles, 31 of which can be accessed free at: http://learnmem.cshlp.org/content/22/11/567.full.html\#ref-list-1

Creative This article, published in Learning \& Memory, is available under a Creative Commons Commons License (Attribution-NonCommercial 4.0 International), as described at License http://creativecommons.org/licenses/by-nc/4.0/.

Email Alerting Receive free email alerts when new articles cite this article - sign up in the box at the Service top right corner of the article or click here. 